\title{
NOTAS DE INVESTIGACIÓN. LA OPINIÓN PÚBLICA EN LA RIOJA. APROXIMACIÓN A NUESTRA EPIDERMIS SOCIAL.
}

\author{
Carmen RUIDÍAZ GARCÍA \\ Profesora Titular E.U. INTERINA De TRABAjO SOCIAL Y SERViCiOS SOCIALES \\ UNIVERSIDAD DE LA RIOJA
}

s $u$ m a r i o

I. Anotaciones sobre el concepto de opinión pública. 2. Aspectos metodológicos de la investigación. 3. Resultados de la investigación. 3.I. Relaciones personales y perfil psicosocial de los riojanos. 3.2. Situación socio-económica y política de la región. 3.4. Mirada prospectiva. 4. A modo de síntesis. 5. Bibliografía.

$\mathrm{r}$ e $\mathrm{s}$ u $\mathrm{m}$ e $\mathrm{n}$

Estas notas de investigación constituyen un retrato - una imagen visual - de las actitudes, opiniones y representaciones sociales que tienen los riojanos sobre sí mismos y la realidad social, económica y política de su Comunidad Autónoma, un retrato extraído de los resultados porcentuales de la encuesta diseñada e implementada para tal fin en la primavera de 2003 (estudio inédito) ${ }^{2}$ y de la explotación de datos secundarios del Centro de Investigaciones Sociológicas.

I. Anotaciones sobre el concepto de opinión pública.

Para comenzar, hay que tener presente que no existe una definición generalmente aceptada de «opinión pública». Sin embargo, los esfuerzos por definir el término han llevado a conclusiones tales como la opinión pública no es el nombre de ninguna cosa, sino una clasificación de un conjunto de cosas.

\footnotetext{
I Carmen Ruidíaz García es profesora de la Universidad de La Rioja, Doctora en Ciencias Políticas y Sociología por la Universidad Complutense de Madrid.

${ }^{2}$ Estudio financiado por el Instituto de Estudios Riojano, Gobierno de La Rioja, Ayudas a la investigación 2002.
} 
Evidentemente, el concepto de opinión pública es un término vago y ambiguo y en gran medida «intangible», puede entenderse, de una parte, como opinión «del público» (sentido subjetivo) y, de otra, como opinión «sobre o acerca de lo público» (sentido objetivo). Quién componga ese «público» de la opinión pública o en qué consista «lo público» como objeto de la opinión pública, son dos preguntas (en realidad, las dos grandes preguntas) a las que hay que intentar responder en cualquier exposición y/o aproximación conceptual al término.

Probablemente, se podría pensar que es posible hablar de un tercer sentido de «opinión pública» que tendría que ver con la idea de «pública» como sinónimo de visible o transparente. La opinión podría entenderse de ese modo, no sólo como la opinión acerca de «lo público» (opinión pública = opinión política o sobre asuntos generales) sino también como opinión visible, que ha sido creada públicamente, sin secretos ni tapujos. Ahora bien, si se piensa, más que ante un nuevo sentido de «opinión pública», se está ante una condición para su existencia, tanto si la contemplamos desde el punto de vista subjetivo como objetivo. Siempre, se trate de la opinión pública como opinión de un público o del público, o se trate de la opinión pública.

Sin embargo, a efectos de este trabajo, aunque la publicidad de una opinión puede ser consecuencia de su interés público, es decir, de que existe un interés subjetivo de los ciudadanos por ese asunto o por lo que opina sobre el mismo (y por eso se publica), es más preciso a la hora de definir a la opinión pública en sentido objetivo hablar de opiniones que interesen objetivamente a la colectividad, esto es, que verse, sobre los asuntos universales, o sobre los problemas políticos, en el sentido profundo y clásico de la expresión.

Por tanto, no constituyen objeto de la opinión pública (en sentido objetivo) las opiniones que traten de los agravios entre cualesquiera personas privadas si no entrañan importancia para el conjunto de la ciudadanía. Público es aquello que influye en la vida política, es decir, la opinión pública no es la opinión privada.

Este es, además el significado que la mayoría de los diccionarios políticos, jurídicos o sociológicos atribuyen al concepto de opinión pública un sentido objetivo. Por ejemplo, en términos jurídicos y/o políticos la opinión pública es todo conocimiento o juicio en virtud de éste que el pueblo que constituye un Estado determinado tiene respecto a los órganos políticos y de gobierno y a las cuestiones que le atañen de manera directa e indirecta; de la misma forma, desde la Sociología se le atribuye al término de opinión pública una importancia política (sin olvidarse del referente cultural), especialmente cuando se le interpreta como actor que representa a los ciudadanos en una democracia; en este sentido, la opinión pública vota, interroga, demanda, aprueba las propuestas o decisiones del poder político3.

\footnotetext{
3 Recientemente, Bouza (2004) hacia unas interesantes apreciaciones sobre el concepto de opinión pública. Para él, en las rutinas y tradiciones académicas sobre la Opinión pública se han mezclado casi siempre dos conceptos distintos: el político y el sociológico. Y continua, la tradición jurídico-política ha oscilado entre una concepción legitimadora de la democracia entendida como régimen de opinión o garantía de democracia y, la tradición sociológica, que nace alrededor de la emergencia de las encuestas y de la Public Opinión Quarterly.
}

Por su parte, Monzón (I987) ha realizado una importante contribución aclaratoria sobre esta cuestión. El hombre razona sus ideas - comenta -, pero también racionaliza sus impulsos, tendencias, intereses y necesidades, y ambas funciones (razonamiento y racionalización) pueden ser verbalizadas y expresadas colectivamente, de lo que se deduce que la opinión pública incluye ambas dimensiones: la racional y la irracional, de forma que la opinión pública exige, ordena, amenaza, aplaude, etc.

Redur 4 / 2006 
Diría más, la opinión pública es uno de los ingredientes indispensables del sistema democrático de gobierno. Todos los gobiernos, con más o menos grado de desarrollo democrático, incluso claramente no democráticos, han pretendido estar gobernando de acuerdo con la opinión pública. En política, hoy nadie se atrevería a afirmar que sus actuaciones o planes de gobierno van en sentido contrario a la opinión pública, sino que todos buscan la legitimidad de estar de acuerdo con ella, o al menos con la de la mayoría. Se trata, por tanto, de una cuestión no controvertida, no contestada, sino unánimemente aceptada, de manera que desde los sistemas más totalitarios a los más democráticos, sus voceros pretenderán estar gobernando de acuerdo con la opinión pública, calificada como mayoritaria.

Por otro lado, en todos los sistemas, la opinión pública suele formarse no a través de un proceso racional y reflexivo mediante el contraste de las diversas informaciones y evaluaciones que hayan recibido, sino que suele forjarse mediante la adopción de evaluaciones prefabricadas que los ciudadanos reciben junto con la información. Es evidente que los ciudadanos forman sus actitudes individuales a partir de los agentes de socialización, como se ha reconocido desde los pioneros de la sociología, es decir, a través de la familia, el grupo de juego infantil, la escuela, la iglesia o confesión a la que se pertenezca o por citar otro ejemplo y, más modernamente, a través de los medios de comunicación.

De cualquier forma, lo importante es saber quién mide y cómo medir las corrientes y estados de opinión y también qué pueden hacer los diferentes actores políticos y sociales con esas mediciones, con esos conocimientos sobre la opinión pública, nuestra «epidermis social» ${ }^{4}$.

2. Aspectos metodológicos de la investigación ${ }^{5}$.

El objetivo básico del estudio realizado en el año 2003 consistía en retratar las imágenes y representaciones sociales que los ciudadanos de la Comunidad Autónoma de La Rioja tienen sobre su realidad social, económica y política. Un objetivo que tiene como foco de interés tres grandes áreas temáticas ${ }^{6}$ : a. Análisis de las relaciones personales y perfil

\footnotetext{
${ }^{4}$ En este sentido, no tengo inconveniente en afirmar que, apoyándome en las reflexiones de Bouza (2004), para mí la Opinión pública son los resultados de encuesta: agregado numérico de conductas verbales públicas en tanto que respuestas a preguntas en un cuestionario. Esta es una de las herramientas de trabajo del Sociólogo desde la que vamos conociendo relativamente bien las conductas sociales de los hombres que vivimos en sociedad. Hoy por hoy, no se conoce procedimiento más preciso de conocimiento de la opinión (popular, general, pública), aunque sabemos también de sus limitaciones que es preciso suplir con estudios paralelos

${ }^{5}$ La Comunidad Autónoma de La Rioja es un lugar especialmente interesante para abordar un estudio en profundidad sobre las actitudes y opiniones de los ciudadanos sobre cuestiones de interés general, y digo esto porque en los últimos años se ha producido un desarrollo socioeconómico sin precedentes. La sociedad riojana de hoy es distinta de la de ayer y, presumiblemente, será distinta de la de mañana.

Situada en el corredor abierto entre el País Vasco y Cataluña, La Rioja se beneficia del extraordinario dinamismo del eje del Ebro, a lo largo del cual se concentran población y recursos financieros y se desplazan importantes flujos de mercancías y de personas. La Rioja constituye un modelo muy perfeccionado de lo que se conoce por economías de situación.

${ }^{6}$ En la investigación se abordó también un cuarto foco: Actitudes políticas, voto e identificación ideológica y simpatías políticas.
} 
psicosocial de los riojanos: sentimiento de felicidad, obstáculos a la felicidad, tolerancia y confianza en los demás, los derechos más importantes para los riojanos. b.- Situación socioeconómica y política de la región: percepción de la situación política, percepción de la situación económica, percepción de los problemas de la Región. c.- Funcionamiento de las instituciones: valoración de la gestión del Gobierno Regional.

Para conseguir estos objetivos realicé un estudio exploratorio y otro descriptivo. El estudio exploratorio consistió, básicamente, en la revisión de la información secundaria del Centro de Investigaciones Sociológicas (encuestas de opinión) con referencia expresa a la Comunidad Autónoma de La Rioja ${ }^{7}$. El estudio descriptivo consistió en realizar una encuesta de opinión pública (primavera 2003) a una muestra significativa en la Comunidad Autónoma de La Rioja.

La fiabilidad y validez de los datos analizados en la encuesta desarrollada en la primavera de 2003 queda garantizada sobre la base de los siguientes recursos metodológicos: a.- Empleo de una muestra representativa para la Comunidad Autónoma de La Rioja. b.- Estandarización de gran parte de las preguntas del cuestionario con las de otras investigaciones nacionales e internacionales, con legitimidad científica asegurada. c.Evaluación de las diferencias entre los resultados finales de la encuesta (primavera 2003) y otras referencias objetivas externas y publicadas, siempre que existan datos contrastables. d.- Segmentación sociodemográfica y actitudinal de los datos de encuesta, en función de las diferencias estadísticamente significativas entre diversos colectivos sociales. Los factores diferenciadores básicos considerados son el sexo, la edad, nivel de estudios y situación laboral. e.- Comparaciones espaciales y temporales con datos de otros estudios locales, nacionales.

Ficha técnica. La recogida de información se realizó entre los meses de abril y mayo de 2003 a través de entrevista individual mediante cuestionario estructurado y cerrado a una muestra representativa de la población de la Comunidad Autónoma de La Rioja.

La muestra (universo del estudio), dirigida a una población de ambos sexos, de i8 y más años, residentes en la Comunidad Autónoma de La Rioja, se realizó por un procedimiento de muestreo polietápico, con selección de las unidades primarias (secciones) de forma aleatoria proporcional, y de las unidades últimas (individuos) por cuotas de sexo y edad en el municipio de Logroño. De las 2I8 entrevistas previstas inicialmente pudimos realizar 200.

La delimitación temática del cuestionario (variables de estudio y redacción de preguntas) fue el resultado de una etapa previa que implicó la recogida y análisis de documentación propia y del Banco de datos del Centro de Investigaciones Sociológicas.

7 Estudios consultados: Centro de Investigaciones Sociológicas www.cis.es E. I.573; E. I.703; E. 2.286; E. 2.334; E. 2.4OI; E. 2.402; E. 2.405; E. 2.442; E. 2.455; E. 2.483; E. 2.496; E. 2.53I. 
3. Resultados de la investigación. 3.I- Relaciones personales y perfil psicosocial de los riojanos.

Sentimiento de felicidad

Cuadro i. En líneas generales, ¿diría usted que es ...? (en porcentajes)

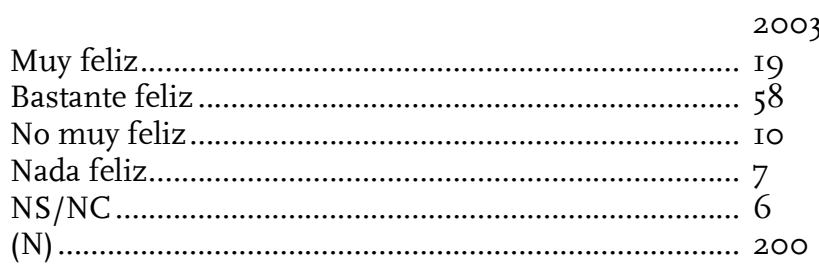

Fuente : Encuesta propia (2003)

En el año 2003 el iو \% de los ciudadanos de la Comunidad Autónoma de La Rioja manifiestan ser muy felices y el $58 \%$ bastante felices, un io \% no es muy feliz y únicamente $7 \%$ señala no ser nada feliz. Agregando los porcentajes de aquellos que se sienten muy felices o bastante felices, se puede señalar que los jóvenes son los más felices y el grado de felicidad desciende ligeramente a medida que aumenta la edad. Los parados son los que manifiestan ser menos felices, uno de cada cinco parados se declara no muy feliz y, por último, el sentimiento de felicidad aumenta a medida que se asciende en la clase social sentida.

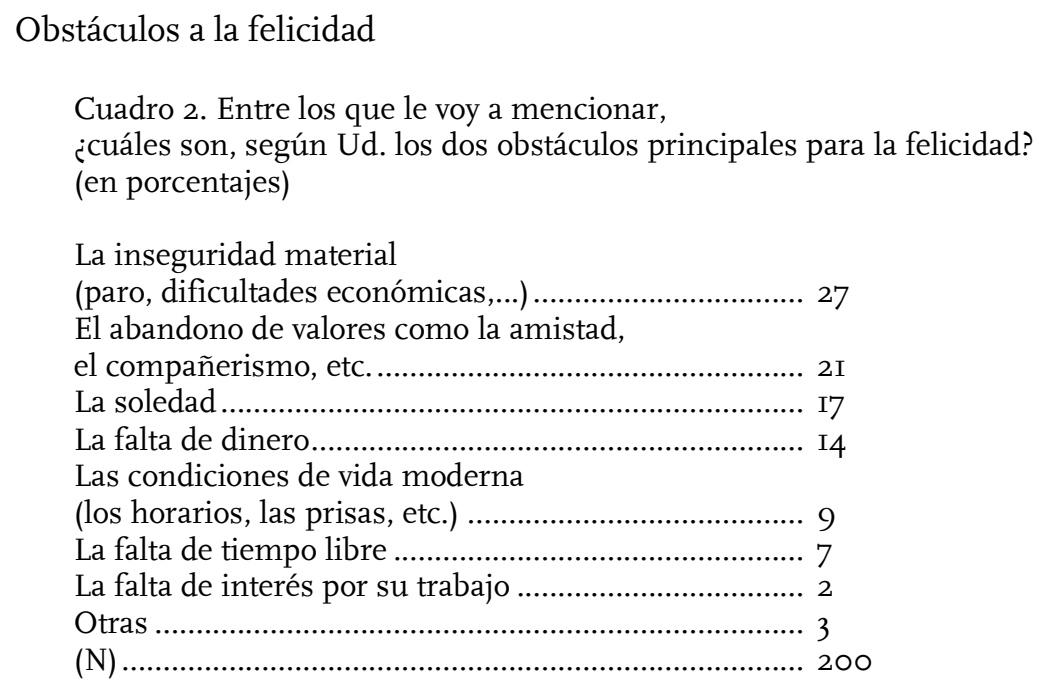

Fuente: Encuesta propia (2003)

Al preguntar a los entrevistados por los principales obstáculos para la felicidad, los datos de encuesta indican que para los riojanos un obstáculo importante para conseguir la felicidad es la inseguridad material, es decir, el paro, las dificultades económicas. Asimismo, el abandono de valores como la amistad, el compañerismo o la soledad son mencionados también como obstáculos 
importantes, mientras que la falta de tiempo libre o el desinterés por el trabajo, son items escasamente significativos ${ }^{8}$.

\section{Confianza en los demás y tolerancia}

Cuadro 3. En general, ¿cree que se puede confiar en la gente o que hay que ir con cuidado? (en porcentajes)

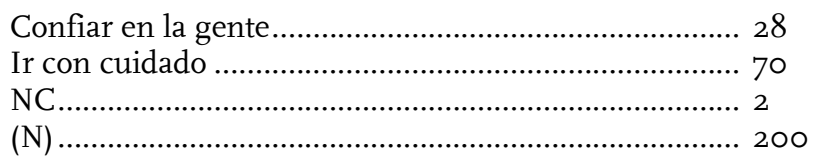

Fuente: Encuesta propia (2003).

Un $70 \%$ de los riojanos consideran que en las relaciones con los otros hay que ser cuidadoso. Ahora bien, si esta misma cuestión - confianza en los demás se analiza en función de la edad, los datos de encuesta indican que los jóvenes son más confiados que los adultos.

En cuanto a la tolerancia, los riojanos se sitúan en una actitud moderada (ver cuadro 4).

Cuadro 4. ¿Le molestaría mucho o bastante, poco, nada tener como vecinos a ...? (en porcentajes)

\section{Mucho o bastante}

Personas alcohólicas ................................................... 35

Personas que estuvieron en la cárcel ........................ 3I

Personas de raza diferente (gitanos, negros) ................ 27

Personas con problemas psíquicos ............................. 25

Personas de extrema derecha ...................................... I5

Personas con SIDA .................................................. 2I

Homosexuales .......................................................... 24

- El abandono de valores como la amistad o el compañerismo es más indicado por los riojanos, los jóvenes (su mención aumenta a medida que desciende la edad), los estudiantes (que le conceden tanta importancia como a la inseguridad material) y las personas de clase social alta.

- $\quad$ La soledad es más mencionada por riojanos mayores de 64 años, encuestados que la sitúan por encima de la inseguridad material. Las personas autocalificadas de clase social alta lo señalan en mayor medida que las de clase media o baja.

- La falta de dinero también es un obstáculo para conseguir la felicidad y es mas mencionada a medida que disminuye la clase social.

- La inseguridad material como obstáculo a la felicidad es más señalada por los riojanos, los de 30-64 años y los parados, y menos por los jubilados y mayores de 64 años. Las personas de clase social media o baja también lo mencionan más que las de clase alta.

Redur 4 / 2006 
Personas de extrema izquierda................................. I7

Inmigrantes, trabajadores extranjeros2 ..................... 8

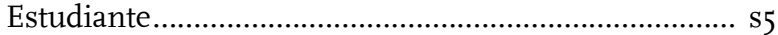

Familias numerosas................................................. 8

Madres solteras ................................................... I4

Fuente: Encuesta propia (2003)

Los derechos más importantes para los riojanos

Cuadro 5. Para cada uno de los siguientes derechos y libertades, ¿’podría decirme, por favor, si piensa que deberían ser respetados en todas las circunstancias o si depende de las circunstancias? (en porcentajes)

La Rioja Total U.E.

El derecho a la educación y a la formación

En todas las circunstancias ----------------------------- 97

Depende de las circunstancias ...................................... 2

NS/NC ....................................................................... I

El derecho a la vivienda

En todas las circunstancias......................................... $96 \quad 83$

Depende de las circunstancias .................................. $3 \quad$ I4

NS/NC.

2

Igualdad ante la ley

En todas las circunstancias................................................. 94

Depende de las circunstancias ......................................... 4

49

$\mathrm{NS} / \mathrm{NC}$

El derecho a la protección legal contra la discriminación

En todas las circunstancias............................................... 93 $93 \quad 83$

Depende de las circunstancias ......................................... 3

El derecho de asilo en caso de persecución política

En todas las circunstancias

Depende de las circunstancias

La libertad religiosa y la libertad de conciencia

En todas las circunstancias

La libertad de expresión

En todas las circunstancias...

Depende de las circunstancias 90 79 
$\mathrm{NS} / \mathrm{NC}$

2

La libertad de asociación

En todas las circunstancias

Depende de las circunstancias

NS/NC

Fuente: Encuesta propia (2003) y Eurobarómetro 47 (I997). Los datos del Eurobarómetro corresponde a ciudadanos de I5 y más años.
3

60

34

- Derechos sociales. Más de nueve de cada diez ciudadanos riojanos defienden que siempre, en todas las circunstancias, debería respetarse el derecho a la educación y la formación, así como el derecho a la vivienda. La segmentación por colectivos no presenta diferencias significativas. Cifras muy similares a las observadas en la Unión Europea, donde los ciudadanos opinan que los derechos sociales deberían ser respetados en todas las circunstancias.

- Derechos jurídico-políticos. Por lo que se refiere a los derechos jurídicopolíticos los datos indican que el $94 \%$ de los ciudadanos riojanos defienden que la igualdad ante la ley deber ser respectada en todas las circunstancias y un porcentaje similar (93\%) cree que también debería respetarse siempre el derecho a la protección legal contra la discriminación. Por su parte, el derecho de asilo en caso de persecución política es defendido en todas las circunstancias por el $74 \%$ de la ciudadanía riojana. Las personas de más de 64 años y las que no alcanzan los estudios primarios presentan mayor nivel de no respuesta que el resto a estas cuestiones, y más en lo relativo al respeto del derecho de asilo en caso de persecución política. Por último, la defensa en todas las circunstancias de estos derechos jurídico-políticos está más extendida en La Rioja que en la media de la UE.

- Garantía de libertades. El 93 \% de los ciudadanos riojanos cree que la libertad religiosa y la libertad de conciencia deben ser respetadas en todas las circunstancias. También un $90 \%$ cree que siempre debería respetarse la libertad de expresión. Y un 86 \% defiende la libertad de asociación para todas las circunstancias. Las opiniones son muy similares entre hombres y mujeres y grupos de edad. Asimismo, nuevamente los riojanos defienden más el respeto a este tipo de libertades en todas las circunstancias que los ciudadanos de la U.E.

3.2.Situación socio-económica y política de la región.

La Rioja es un espacio geográfico y social equilibrado. Su alto nivel de vida, sus buenas comunicaciones y servicios, la calidad de sus gentes, etc. le convierten en un espacio privilegiado donde se asienta, y refuerza, la cultura del «buen vivir» ${ }^{9}$. Los

\footnotetext{
${ }^{9}$ Por ejemplo, y según datos del Anuario Estadístico de la Comunidad Autónoma de La Rioja, en el cuarto trimestre de 2002 la tasa de paro en La Rioja era de 6,10 \%, mientras que en España la cifra
}

Redur 4 / 2006 
datos de encuesta refuerzan este estereotipo, esta realidad. Así, a la hora de valorar la situación global de la región un $29 \%$ de los riojanos consideran que es buena o muy buena y un $20 \%$ mala o muy mala.

En cuanto a la valoración de la situación política de la Comunidad Autónoma de La Rioja es moderadamente optimista. El $29 \%$ de los entrevistados la califica de una forma positiva y para un $40 \%$ merece el calificativo de regular y un $20 \%$ la califican de mala o muy mala.

En la encuesta de 2003, llama la atención que la valoración más positiva sobre la situación política de La Rioja corresponde a los encuestados que se han identificado ideológicamente en posiciones de derecha o centro-derecha. En cuanto al sexo de los entrevistados, la valoración de los hombres es mucho más optimista que la de las mujeres. Por último, al considerar la edad de los encuestados, se observa una mejor valoración de la situación política de la región a medida que se avanza en los tramos de edad.

En términos generales, la situación económica de la Comunidad Autónoma es valorada positivamente. En la encuesta se contempla que existe una relación positiva entre el mayor optimismo en la valoración económica de la Comunidad y una posición ideológica de derecha, al igual que la valoración más positiva y/o optimista corresponde a los hombres. En cuanto a la edad de los entrevistados, no se observa una relación significativa entre esta variable y la valoración de la situación económica.

Si comparamos estos datos retrospectivamente (ver cuadro 6), la percepción de los riojanos no ha cambiado mucho, sigue siendo positiva.

\begin{tabular}{|c|c|c|c|}
\hline & Situación política & Situación económica & Situación general \\
\hline Muy buena & $\mathrm{I}, 9$ & $3, \mathrm{I}$ & 4,5 \\
\hline Buena & 38,9 & 48,6 & 57,3 \\
\hline Regular & 36,7 & 33,8 & $29, I$ \\
\hline Mala & 8,7 & 8,7 & 4,8 \\
\hline Muy mala & $3, \mathrm{I}$ & 2,2 & $\mathrm{I}, \mathrm{O}$ \\
\hline N.S. & IO,I & 3,4 & 3,3 \\
\hline N.C. & 0,5 & 0,2 & \\
\hline
\end{tabular}

Fuente: CIS. Situación política y situación económica E. 2286, situación general E. 2334.

Ahora bien, en La Rioja también hay dificultades, problemas y así lo perciben los

asciende a II,45 \%. La renta familiar disponible en La Rioja en el año 200I era de II3,3I, frente al I00,00 de la media Nacional o el II3,56 de la Comunidad Navarra o, por citar un último ejemplo, 83,20 de la Comunidad de Murcia. El precio medio del metro cuadrado de la vivienda (en euros) en el año 200I en La Rioja era de 926,29, en España de I.०46,76. 
riojanos.

Cuadro 7. En su opinión, ¿cuál es el problema más importante que tiene la Comunidad Autónoma en estos momentos? (en porcentajes)

Primer problema

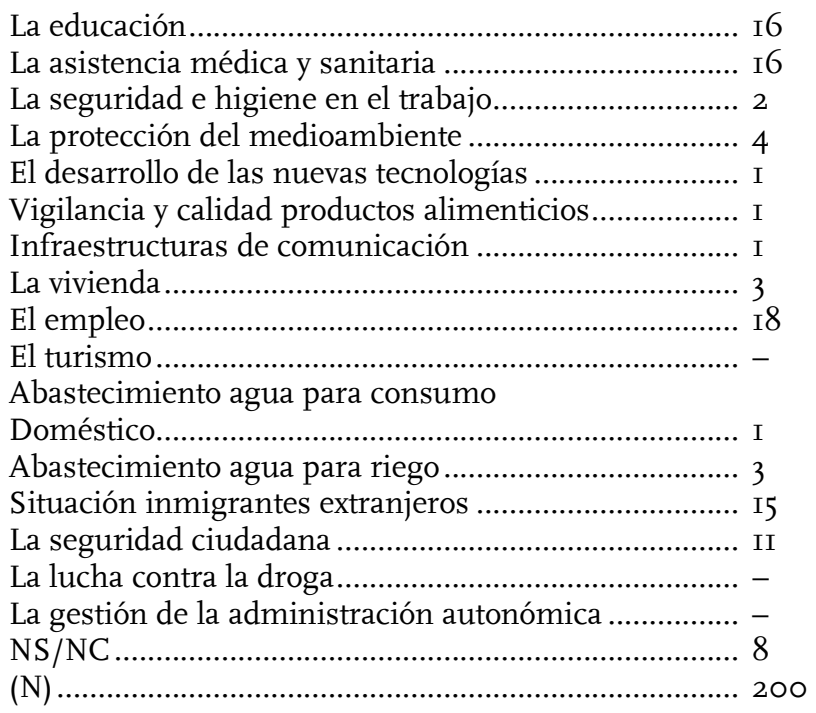

Fuente: Encuesta propia (2003)

Los entrevistados consideran que el principal problema de la Comunidad Autónoma es el empleo con un i8 \%, la educación, asistencia sanitaria, la seguridad ciudadana y la situación de los inmigrantes extranjeros son situaciones problemáticas que preocupan a los riojanos en primera opción.

La identificación ideológica del entrevistado matiza ligeramente la consideración sobre la importancia del principal problema de la Comunidad: el empleo. En este sentido, los entrevistados que se sitúan más a la izquierda de la escala ideológica ponderan en mayor medida la importancia del problema. En cuanto al problema de la situación de los inmigrantes extranjeros, aunque es un asunto considerado importante por todos los entrevistados, es una preocupación hacia la que se encuentran especialmente sensibilizados los situados ideológicamente en centro izquierda en la escala ideológica. Y, por último, todos los entrevistados, sin distinción ideológica, se sienten preocupados por la seguridad ciudadana $^{\mathrm{IO}}$.

Io «Paro, inmigración e inseguridad ciudadana, principales preocupaciones de los riojanos. Los encuestados creen que los problemas de la seguridad ciudadana y de la vivienda son más graves en La Rioja que en el conjunto nacional». Fuente: Diario de La Rioja, 20 de noviembre de 2002.

Redur 4 / 2006 
3.3. Funcionamiento de las instituciones y valoración política del Gobierno Autonómico.

En la vida de los pueblos existen «momentos estelares». En La Rioja, uno de esos momentos es el que al constituirse en Comunidad Autónoma. El proceso autonómico de La Rioja no ha sido fácil. A lo largo de todo su recorrido se ha pasado por momentos de esperanza y de zozobra y se ha superado gracias a una voluntad común de seguir adelante, muchas veces supeditando el criterio partidista al sentimiento regional. Y es así como, paso a paso, fueron superándose etapas que van desde que el Ayuntamiento de Logroño tomó el primer acuerdo para que se iniciase el proceso, hasta que el Rey sancionase el Estatuto de Autonomía de La Rioja como nueva Ley Orgánica. Un hito histórico que ha modificado sustancialmente el curso de la Historia de esta tierra - La Rioja.

Hoy estamos en disposición de evaluar el proceso y también de ofrecer pinceladas de realidad sobre el grado de legitimidad de dicho proceso, es decir, sobre la consolidación de la Autonomía de La Rioja (una de las Comunidades más pequeñas y menos poblada de cuantas configuran el mapa autonómico español).

Los encuestados por el C.I.S. en I987 (E. I573, encuesta preelectoral municipales y autonómicas) valoran muy positivamente la creación y desarrollo del Estado de las Autonomías y consideran, por amplia mayoría, que en el caso de La rioja, ha sido favorable para el desarrollo social y político de la Comunidad.

\begin{tabular}{|c|c|c|c|c|c|c|}
\hline & $(\mathrm{N})$ & $\begin{array}{l}\text { Ha mejorado } \\
\text { mucho }\end{array}$ & $\begin{array}{l}\text { Ha mejorado } \\
\text { algo }\end{array}$ & $\begin{array}{l}\text { No ha } \\
\text { cambiado }\end{array}$ & $\begin{array}{l}\text { Ha empeorado } \\
\text { algo }\end{array}$ & $\begin{array}{l}\text { Ha empeorado } \\
\text { mucho }\end{array}$ \\
\hline \multicolumn{7}{|l|}{ SEXO } \\
\hline Hombre & 547 & 5,6 & 53,7 & 22,3 & 7,7 & $\mathrm{I}, \mathrm{I}$ \\
\hline Mujer & 570 & 5,4 & 50,8 & $2 \mathrm{I}, 7$ & 5,5 & $\mathrm{I}, \mathrm{I}$ \\
\hline TOTAL & III 8 & 5,5 & 52,3 & 22,0 & 6,6 & $\mathrm{I}, \mathrm{I}$ \\
\hline \multicolumn{7}{|l|}{ EDAD } \\
\hline $18-25$ & 192 & 5,8 & 62,2 & 20,9 & $4, \mathrm{I}$ & $\mathrm{I}, 4$ \\
\hline $26-40$ & 288 & 4,7 & 56,8 & 22,4 & 5,3 & $\mathrm{I}, \mathrm{O}$ \\
\hline $4 \mathrm{I}-64$ & 442 & $6, \mathrm{I}$ & 50,2 & $2 \mathrm{I}, 7$ & 8,6 & $\mathrm{I}, 2$ \\
\hline $6_{5}$ y más & I96 & 4,8 & $4 \mathrm{I}, \mathrm{O}$ & 22,9 & 6,4 & 0,7 \\
\hline TOTAL & III7 & 5,4 & 52,4 & 22,0 & 6,6 & $\mathrm{I}, \mathrm{I}$ \\
\hline
\end{tabular}

Fuente: CIS, E. I573

Unos datos que estaban en consonancia con el grado de satisfacción que manifestaban tener los ciudadanos de otras Comunidades Autónoma. E. I573: Castilla-La Mancha 52, Cataluña 48, Navarra 43, Cantabria 37, Andalucía 34, Canarias 26. Estos datos se ven reforzados por el alto grado de satisfacción que manifiestan tener los riojanos con el funcionamiento de la Comunidad Autónoma. 
Años después la percepción de los riojanos sobre el desarrollo y consolidación de la Autonomía regional sigue siendo muy bien valorada por los ciudadanos. En este sentido, y en términos globales, en el año 2003 la valoración de la gestión del Gobierno de la Comunidad es positiva en todos los grupos de edad; ahora bien, destacan los de derecha o centro-derecha de la escala ideológica, prácticamente en la misma medida en que es valorado negativamente entre los entrevistados situados en la izquierda y el centro-izquierda de dicha escala. En función del nivel de estudios de los encuestados, las posturas sobre la gestión del Gobierno autonómico, son ligeramente más críticas entre los entrevistados con un nivel de estudio medio-alto.

\subsection{Mirada prospectiva.}

A lo largo del desarrollo de la democracia han nacido y crecido generaciones de jóvenes electores que se van a incorporar al inmediato futuro con unas condiciones de socialización completamente distintas que las de sus predecesores. En estos casos, la memoria histórica de la dictadura se difumina, tanto para valorarla positivamente como negativamente y la línea divisoria entre franquismo y postfranquismo es cada vez más tenue.

Quizás esta sea la razón, en la primavera de 2003, dos tercios de los ciudadanos riojanos piensan que nuestros hijos y nietos tendrán una vida mejor que la de nuestros padres y abuelos. Algo más de la mitad piensa que habrá más gente pobre y socialmente excluida. También son algo más de la mitad los que piensan que el paro seguirá en los mismos niveles que hoy en día. Un $37 \%$ de los riojanos creen que en este siglo XXI habrá menos guerras y menos violencia en el mundo, un $23 \%$ de los riojanos cree que el siglo XXI será peor que el siglo XX. Cabe destacar que las personas de menos de 46 años son más escépticas respecto a lo que nos deparara el siglo XXI que quienes superan esa edad. Las personas de izquierdas también son más escépticas respecto al futuro. Sin embargo, y a pesar de estos datos, los riojanos confían más que la media europea en que nuestros sucesores vivirán mejor que nuestros antepasados. Igualmente los riojanos también están menos convencidos que los europeos de que el paro se vaya a mantener en los niveles actuales.

Cuadro 8. Pensando en el futuro, dígame si está Ud. más de acuerdo o más bien en desacuerdo con cada una de las siguientes afirmaciones (en porcentajes)

\section{La Rioja Total U.E.}

En el siglo XXI nuestros hijos y nietos tendrán una vida mejor que la de nuestros padres y abuelos

En todas las circunstancias

Depende de las circunstancias

En el siglo XXI habrá más gente pobre y socialmente excluida 


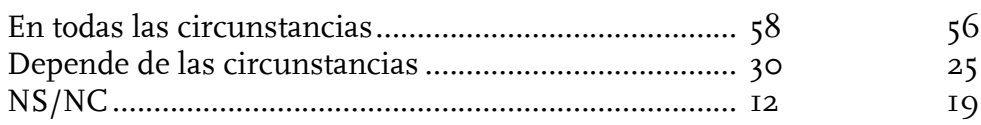

En el siglo XXI habrá tanto paro como hoy en día En todas las circunstancias..........................................5 54

Depende de las circunstancias .................................. 34 I9

NS/NC .............................................................. I2

En el siglo XXI habrá menos guerras y menos

violencia en el mundo

En todas las circunstancias ........................................ 37

Depende de las circunstancias .................................. 49

NS/NC ............................................................. I4 23

El siglo XXI será peor que este siglo

En todas las circunstancias........................................ 23

Depende de las circunstancias ................................... 52

NS/NC .................................................................. 25

Fuente: Encuesta propia (2003) y Eurobarómetro 47 (I997). Los datos del Eurobarómetro corresponde a ciudadanos de i5 y más años.

4. A modo de síntesis.

La opinión pública no es sólo un fenómeno colectivo entre otros muchos fenómenos de la vida social sino que constituye también una institución fundamental del sistema democrático de gobierno.

En mi estudio, o la hora de hacer balance, solo queda resaltar que los riojanos se sienten satisfechos, felices o relativamente felices con su vida y su Comunidad Autónoma (en lo social, político y económico) y, también, en La Rioja se ha producido un proceso de institucionalización y cristalización de las organizaciones e instituciones democráticas. Los datos de encuesta (datos primarios y secundarios) permiten afirmar que las instituciones democráticas tienen un alto grado de legitimidad en la sociedad riojana e, igualmente, permiten sostener que se han generado hábitos y comportamientos político democráticos entre los ciudadanos de La Rioja.

5. Bibliografía.

AgUiAR, F. (I99I): Intereses individuales y acción colectiva, Madrid, Editorial Pablo Iglesias.

Almond, G. y VerbA, S. (I970): La cultura cívica, Madrid, Euroamérica.

(1989): The Civic Culture Revisited. An analytic study, London, Sage.

Arblaster, A. (I992): Democracia, Madrid, Alianza Editorial.

BENEDiCTO, J. (I993): «Espectadores o actores potenciales?. El debate sobre los sistemas de creencias políticas de los ciudadanos», Revista de Estudios Políticos, 80, pp.27i.295.

BouZA, F.(2004): «Desventajas de un concepto claro y distinto: opinión publica», en VV.AA., Reflexiones sociológicas. Libro homenaje a José Castillo. 
CASTELS, F.G. (1978): The social democratic image of society, Londres, Routledge and Kegan Paul.

CoOp, HAMpton y Roemer (I993): The idea of democracy, Cambridge, Cambridge University Press.

DAHL, R. (I992): La democracia y su críticos, Barcelona, Paidós.

DAVIS, J. (I986): «British and American Attitudes: Similarities and Contrasts», en R. Jowell, S. Witherspoon y L. Brook (comps.), British Social Attitudes, Aldershot, Gower.

DOWSE, R. y HugheS, J. (I986): Sociología política, Madrid, Alianza Universidad.

HABERMAS, J. (I98I): Historia y crítica de la opinion pública. La transformación structural de la vida pública, Barcelona, Gustavo Gilo.

HeLD, D. (1989): Political theory and the modern state, Cambridge, Polity Press.

(I992): Modelos de democracia, Madrid, Alianza Editorial.

LINZ, J.J. (I985): De la crisis de un Estado unitario al Estado de las Autonomías, en F.

(1987): La quiebra de las democracias, Madrid, Alianza Editorial.

(I988): Legitimacy of democracy and the socio-economic system, en M. DoGAN (comp.), Comparing pluralist democracies: strains on legitimacy, Boulder, Westview.

(I990): «Reflexiones sobre la sociedad española», en SALVADOR Giner (ed.), España, Sociedad y Cultura, Madrid, Espasa Calpe.

Lippmann, W. (1965): Public Opinion, Nueva York, The Free Press.

LóPEZ-ARANGUREN, E. (I983): La conciencia regional en el proceso autonómico español, Madrid, Centro de Investigaciones Sociológicas.

LÓPEZ PINTOR, R. (198I): Las bases sociales de la democracia en España, Madrid, Fundación Humanismo y Democracia.

(I982): La opinión pública del franquismo a la democracia. Madrid, CIS.

Montoro, J. R. y TorCAL, M. (I990): «La cultura política de los españoles: pautas de continuidad y cambio». Rev. Sistema 99.

MonZón, C. (1987): La Opinión Pública. Teorías, concepto y método, Madrid, Tecnos.

SennetT, R. (1978): El declive del hombre público, Barcelona, Ed. Península. 